Cinémas

Revue d'études cinématographiques

Journal of Film Studies

\title{
Le cinéma des premiers temps à Montréal et l'institution du théâtre
}

\section{Jean-Marc Larrue}

Volume 6, numéro 1, automne 1995

Le cinéma muet au Québec et au Canada : nouveaux regards sur une pratique culturelle

URI : https://id.erudit.org/iderudit/1000962ar

DOI : https://doi.org/10.7202/1000962ar

Aller au sommaire du numéro

Éditeur(s)

Cinémas

ISSN

1181-6945 (imprimé)

1705-6500 (numérique)

Découvrir la revue

Citer cet article

Larrue, J.-M. (1995). Le cinéma des premiers temps à Montréal et l'institution du théâtre. Cinémas, 6(1), 119-131. https://doi.org/10.7202/1000962ar
Résumé de l'article

Cet article propose un tableau précis des liens étroits qui unirent le cinéma des premiers temps à l'institution théâtrale montréalaise existante. À cette époque, le cinéma joue un rôle central dans les stratégies d'émergence du théâtre francophone qui s'éloigne alors du théâtre anglophone, son rival issu de la ville de New York, tout autant qu'il joue un rôle d'attrait dans un contexte de vive concurrence. Non seulement cela donne-t-il lieu à des scènes mixtes (théâtrales et cinématographiques), mais également à une interprétation plus profonde de deux arts qui aboutit aux premières manifestations d'un cinéma-théâtre québécois. 


\title{
Le cinéma des premiers temps à Montréal et l'institution du théâtre
}

\author{
Jean-Marc Larrue
}

\section{RÉSUMÉ}

Cet article propose un tableau précis des liens étroits qui unirent le cinéma des premiers temps à l'institution thêâtrale montréalaise existante. À cette époque, le cinéma joue un rôle central dans les stratégies d'émergence du théâtre francophone qui s'éloigne alors du théâtre anglophone, son rival issu de la ville de New York, tout autant qu'il joue un rôle d'attrait dans un contexte de vive concurrence. Non seulement cela donne-t-il lieu à des scènes mixtes (théâtrales et cinématographiques), mais également à une interprétation plus profonde de deux arts qui aboutit aux premières manifestations d'un cinéma-théâtre québécois.

\section{ABSTRACT}

This article presents a detailed portrait of the relationships between early cinema and theatrical institutions in Montréal. Early cinema played a key role in the strategies of emergence of the city's Francophone theatre, then in the process of distinguishing itself from Anglophone theatre, its New York based rival, as well as acting as an attraction in a context of fierce competition. This led not only to mixed houses, where theatre and cinema shared the stage, but also to a deeper interpenetration of the two art forms, resulting in the first appearance of a dramatic cinema in Québec.

La chronologie des débuts du cinématographe au Québec est à peu près établie. On sait quand et où eut lieu la première 
démonstration - privée - de l'appareil des frères Lumière et on peut suivre, à la trace, les projections subséquentes du cinématographe et de ses concurrents dans différents théâtres et "lieux d'amusement " québécois jusqu'à la Première Guerre mondiale '. Mais notre compréhension des conditions d'émergence de ce qui allait devenir le $7^{c}$ art - et qui n'était encore, au tournant du siècle, qu'une curiosité scientifique —, reste bien parcellaire, voire confuse. La succession étourdissante des appareils de projection, réputés toujours plus perfectionnés, les prétentions d'inventeurs "patenteux" sans cesse plus nombreux, les accusations répétées de plagiat, même de vol, ne contribuent évidemment pas à clarifier la situation. Pourtant, si agaçante qu'elle paraisse pour les historiens, cette confusion ne porte guère à conséquence. Elle découle de facteurs internes, prévisibles et inévitables dès lors qu'une nouvelle pratique s'instaure. Mais justement, le cinéma n'est pas qu'une autre pratique nouvelle; c'est, assez rapidement, un loisir de masse qui, à ce titre, doit composer avec les lois d'un marché hautement concurrentiel et qui doit s'adapter à ce marché pour survivre.

Cet aspect des débuts du cinéma reste assez méconnu en dépit de son importance, en dépit aussi de ses effets sur l'évolution du spectacle cinématographique, particulièrement au Québec. Car, faut-il le rappeler, le cinéma des premiers temps ne s'est pas déployé en plein désert. En 1896, le marché nordaméricain du loisir culturel est déjà bien structuré. Une solide institution du spectacle, centralisée à New York, alimente l'immense majorité des "lieux d'amusement", grands et petits, des États-Unis et du Canada. Or, c'est précisément dans ces lieux que le cinéma effectue sa percée décisive, une percée sans doute moins exceptionnelle qu'on a eu tendance à l'affirmer, mais une percée comparable à celle que connaîtront ou que connurent d'autres genres de spectacles à succès - le vaudeville américain, le muséum, les cabarets - à partir des années 1880 . Toutefois, à la différence de ces derniers, le cinéma perdurera ${ }^{2}$ au point que, très rapidement, il devra créer son propre réseau de distribution. À Montréal, par exemple, il s'écoule à 12 douze ans entre l'initiative des deux agents des frères Lumière et l'avènement de ce qu'il est aujourd'hui convenu d'appeler l'"ère des scopes" (ou 
des premières véritables salles permanentes de cinéma)! Mais le cinéma, surtout le cinéma des premiers temps, n'a pas à se glorifier de ces progrès rapides. Plus opportunistes qu'aventuriers, ses agents ont surtout su profiter des acquis du théâtre, tournant à leur avantage les aléas de la conjoncture.

\section{La filière théâtrale}

Il est de notoriété publique que beaucoup des premiers créateurs de cinéma sont issus de la scène théâtrale et lui doivent leurs premiers succès. Mais la dette du cinéma des premiers temps envers le théâtre ne s'arrête pas là. L'infrastructure dans laquelle il s'épanouit, le public qui l'acclame, la critique qui le salue relèvent également de l'institution thêâtrale, une institution qui, en 1896, éprouve de sérieuses difficultés. La crise économique, qui éclate en 1894 et qui s'étend rapidement à toute l'Amérique du Nord, frappe le marché du loisir de plein fouet. La saison thêatrale 1894-1895 est catastrophique et la saison subséquente s'annonce pire encore. On ne compte plus les établissements qui, à travers tout le continent, sont contraints de fermer leurs portes ou de se recycler en salles de "bal" ou de combats sportifs. Des artistes de renom sont condamnés au chômage et des troupes prestigieuses se dissolvent chaque semaine. Pour conserver leur public appauvri et clairsemé, les agents de théâtre en sont réduits à baisser le prix des billets alors que l'inflation gonfle les coûts de production. La concurrence est féroce, même meurtrière. La situation est si alarmante que six magnats du thêatre nord-américain entreprennent d'unir leurs forces et de mettre au point une stratégie commune afin d'endiguer la crise. Cette initiative historique, qui a pour objectif premier de relancer le marché du théâtre et du spectacle, résulte en la création d'un organisme extrêmement puissant, connu sous le nom de "Trust" ou "Syndicate" des théâtres ${ }^{3}$, dont les ambitions hégémoniques se manifestent rapidement.

La naissance et l'action du Trust n'ont pas d'impact direct ni immédiat sur le cinéma, mais elles créent un contexte qui lui est exceptionnellement favorable. Très vite, en effet, le Trust se heurte aux vélléités d'indépendance de quelques propriétaires influents de troupes et de salles. Regroupés autour des frères 
Jacob, Lee et Sam Shubert, de Syracuse, les "Indépendants» mènent une lutte acharnée au Trust, entraînant ainsi toute l'industrie du thêâtre et du spectacle d'Amérique du Nord dans une lutte commerciale qui s'étendra sur plus de 10 ans. Les deux groupes concentrent leur action sur le théâtre de répertoire ou d'auteur - le legitimate ${ }^{4}$ - , cherchant ainsi à contrôler les établissements les plus prestigieux et les plus importants du continent - tels que l'Académie de musique de Montréal —, qui étaient également considérés comme les plus rentables. Les protagonistes délaissent ainsi sciemment les salles secondaires et presque tout le champ des spectacles de variétés (le non legitimate $)^{5}$.

Obnubilés par la lutte fratricide qu'ils se livrent pendant les dernières années du XIX ${ }^{e}$ siècle et les premières années du $\mathrm{XX}^{e}$ siècle, le Trust et les Indépendants ne perçoivent pas le formidable potentiel que représente le cinéma et n'entreprennent rien pour juguler sa croissance ou pour en tirer parti. Au contraire, ils laissent la voie entièrement libre aux entrepreneurs en cinéma qui n'en attendent pas tant. Ces derniers vont ainsi pouvoir investir les salles secondaires et nouer des relations étroites et durables avec les producteurs de spectacles de variétés.

La crise de l'institution du spectacle n'est pas seule à jouer en faveur du cinéma. L'évolution du goût du public contribue aussi à son succès. L'ère des recettes artistiques éprouvées et des reprises de grands succès tire à sa fin ${ }^{6}$. Les spectateurs ne jurent désormais plus que par l'inédit et la nouveauté, preuve - s'il en fallait! - que la modernité frappe déjà aux portes de l'Amérique. "Notre intérêt pour l'art est passager, notre intérêt pour la nouveauté est permanent ", regrette amèrement et à juste titre le critique du New York Press dans son bilan de la saison thêâtrale 1894-18957. Érigée en véritable culte, la nouveauté devient le maître mot pour toutes les scènes du continent, particulièrement les scènes secondaires, les scènes de variétés entre autres, qui rivalisent d'audace dans le domaine. Lieu privilégié de toutes les expérimentations, elles accueillent le cinéma à bras ouverts, lui réservant d'abord une place modeste entre un astonishing numéro de chiens savants "sous hypnose" et le tour de chant d'une chanteuse de genre dont la voix ne ressemblait 
évidemment à rien de ce que l'oreille humaine n'avait encore entendu! Mais le cinéma va bientôt surpasser en popularité tous les autres numéros de spectacles de variétés - même les plus farfelus et les plus déroutants - au point de les éclipser et de s'affranchir rapidement d'un genre qui l'étouffe. Le loup était entré dans la bergerie et aucun agent de théâtre, pas même le plus perspicace, ne le comprenait.

\section{Montréal dans la tourmente}

Totalement intégrée au marché nord-américain du spectacle, Montréal vit la crise aussi rudement que les autres grandes villes du continent qui jouissent comme elle du statut de one-week$s t a n d^{8}$. Depuis le début des années 1880 , le marché montréalais du spectacle est dominé par deux groupes d'intérêts, le premier est lié au théâtre Royal, le second à l'Académie de musique. En plus du théâtre Royal, John Sparrow et Henry Jacobs contrôlent le Queen's (de Montréal) et cinq autres théâtres dans le nord-est des États-Unis et en Ontario. Quant à l'Académie de musique, elle a acquis au fil des années une solide réputation de théâtre haut de gamme grâce à la gestion avisée de Henry Thomas. C'est elle qui a accueilli toutes les sommités de la scène internationale qui, depuis 1880 , font halte à Montréal, à commencer par Sarah Bernhardt et Henry Irving. Thomas meurt en 1893 et est remplacé à la tête de l'entreprise par son adjoint Frank Murphy qui s'efforce de maintenir la réputation de l'établissement.

Sparrow et Jacobs ne résistent pas longtemps aux pressions du Trust qui menace de les asphyxier en privant de spectacles le petit réseau qu'ils se sont constitué. Aussitôt intégrés au groupe, ils ont pour mission d'éliminer Murphy qui, jaloux de son autonomie et soucieux de perpétuer la tradition instaurée par son prédécesseur, refuse obstinément de se soumettre aux diktats de l'organisation new-yorkaise. La stratégie de Sparrow et Jacobs est simple. Elle consiste à déclasser l'Académie en faisant du Queen's son concurrent direct.

En plus de ces trois géants, la ville dispose de deux autres grandes salles de spectacles en 1896: le théâtre Français et le Monument-National. Le premier cherche encore sa vocation et 
un public — français ou anglais, ou les deux — plus de 10 ans après sa reconstruction et le second, propriété de l'association Saint-Jean-Baptiste de Montréal, se consacre, pour l'essentiel, aux activités des amateurs francophones.

Outre l'Académie et le théâtre Royal, les seules scènes vraiment dynamiques de la ville appartiennent donc à de petits établissements, aux carrières parfois bien éphémères, qui se concentrent boulevard Saint-Laurent et dans les rues avoisinantes. C'est dans ces salles, dont la capacité varie de quelques dizaines à quelques centaines de places, que les Montréalais vont étancher leur soif de nouveauté. Si la rue Sainte-Catherine est en passe de devenir le royaume incontesté du théâtre consacré ${ }^{9}$, le boulevard Saint-Laurent - véritable Off-Broadway avant l'heure - se distingue par son goût de l'original, de l'inédit, bref de l'aventure. Terre d'accueil bénie par les entrepreneurs en tout genre - du spectacle comme des autres commerces —, la Main est évidemment boudée, voire méprisée, par les adeptes du théâtre sérieux. Elle ne représente donc pas un enjeu aux yeux du Trust - puis des Indépendants - et échappe, grâce à cela, au conflit ravageur qui affaiblit les grands théâtres de la ville. Pas étonnant donc que ce soit dans cette artère en continuelle ébullition que le marché du spectacle risque ses expériences les plus hardies, du muséum au cabaret, du burlesque au strip-tease, du concert de big band au concert de jazz. Pas étonnant non plus que ce soit là que Louis Minier et Louis Pupier, les deux envoyés des frères Lumière, choisissent de réaliser, le 27 juin 1896, la première projection cinématographique au pays. Comme pour ajouter à la dimension symbolique de l'événement, ils installent leur projecteur et leur écran dans un ancien muséum, le Gaiety Museum \& Theatorium, pompeusement rebaptisé Palace Theatre ${ }^{10}$.

Petits établissements hybrides, combinant toujours un spectacle de variétés à une quelconque exposition - à prétention scientifique et éducative —, les muséums, qui échappent aux ambitions du Trust, cherchent désespérément à se refaire une vertu. Leur réputation avait été fort malmenée au cours de la décennie précédente et le cinéma leur offre une planche de salut. Il peut, en tant que curiosité scientifique, leur redonner une certaine légitimité ${ }^{\prime \prime}$. 
Après leur "démonstration" à Montréal, les agents des Lumière prennent la route de New York. La chose peut surprendre si l'on considère que c'est dans la métropole américaine que se concentre l'essentiel de l'industrie cinématographique naissante et que se joue son avenir en Amérique. Mais en faisant halte à Montréal, Pupier et Minier suivent simplement le mouvement tracé avant eux par d'innombrables artistes et créateurs français qui rêvaient de triompher aux États-Unis et qui, plus souvent à tort qu'à raison, choisissaient Montréal comme tremplin.

Les conditions dans lesquelles se déroule cette première projection de "vues animées" reste à préciser, mais il est clair que l'importance historique du moment n'échappe à personne et qu'elle permet de minimiser l'inconfort de la salle comme les inconvénients causés par "les trépidations de l'instrument [qui] fatiguaient l'oeil, nuisaient à la netteté de la perception et, parfois, donnaient aux objets cette teinte vague des choses entrevues comme en un rêve ${ }^{12}$ ".

\section{L'accès aux grandes salles}

Le retentissement de l'expérience, plus que sa qualité technique, suscite évidemment l'intérêt d'autres directeurs de petites salles en quête, eux aussi, de l'ultime nouveauté. On aurait donc pu s'attendre que d'autres muséums ou scènes de variétés populaires, comme le parc Sohmer ${ }^{13}$, accueillent à leur tour la "merveille du siècle" et que le cinéma reste cantonné pour quelques années encore au circuit des salles secondaires. Mais il n'en est rien. Ce n'est pas en effet dans un quelconque Dime Museum que le cinéma trouve son asile subséquent, mais dans l'un des deux plus importants théâtres de la ville, le théâtre Royal en l'occurrence. Dès le 28 septembre 1896, soit trois mois presque jour pour jour après la projection inaugurale de Pupier et de Minier, l'établissement annonce à grand bruit la "première exposition en ce pays du Kinématographe", "merveille scientifique de l'univers ${ }^{14}$ ". Le fait qu'un "grand" théâtre comme le théâtre Royal se risque à cette époque à présenter des vues animées est tout à fait inattendu et mérite qu'on s'y attarde, d'autant plus que trois mois après ce coup d'éclat, la presse 
annonce qu'un autre théâtre important de Montréal, le théâtre Français ${ }^{15}$, se lance à son tour dans l'aventure cinématographique grâce au Phantascope d'Edison et à de "nouvelles vues intéressantes ${ }^{16}$ " qu'il projette jusqu'à la fin de l'année. Le mouvement persiste puisque dès les premiers jours de 1897 , le public montréalais est convié à admirer les beautés du Cinémascope ${ }^{17}$ au théâtre Queen's, rien de moins! En quatre mois, le cinéma a ainsi fait son entrée dans trois des quatre établissements de théâtre professionnel les plus importants et les plus prestigieux de la ville. Seule l'Académie de musique échappe encore à sa boulimie.

Cette percée fulgurante et remarquable laisse pantois. La situation montréalaise constitue un précédent! S'il est vrai que le cinéma ne fait que meubler les entractes des programmes de ces grandes salles, il est aussi indéniable que, ce faisant, il gagne considérablement en prestige et, surtout, accroît son rayonnement et son pouvoir d'attraction sur le public. Les trois grands théâtres montréalais accueillent jusqu'à 13000 spectateurs par jour ${ }^{18}$ ! Ainsi, et contrairement à ce qui se passe presque partout ailleurs, le cinéma fait à Montréal ses véritables débuts commerciaux sur des scènes majeures, exclusivement.

La projection régulière de vues animées dans les établissements les plus importants de la ville témoigne bien sûr de l'engouement précoce des Montréalais pour le cinéma. Mais d'autres facteurs expliquent cette situation.

Montréal, rappelons-le, vit rudement la crise qui secoue tout le monde nord-américain du spectacle. Or, en dépit des efforts du Trust et des initiatives de Sparrow et de Jacobs, ses alliés locaux, Murphy résiste et parvient pour un temps à maintenir son Académie de musique en activité. La tactique qui a d'abord consisté à faire du Queen's le rival direct de l'Académie pour mieux la déclasser n'ayant pas apporté les résultats escomptés, le Trust et ses agents usent de moyens plus sûrs et plus expéditifs. En mars 1896, le Service des incendies de la ville de Montréal entreprend en effet une inspection impromptue et anormalement minutieuse des lieux. Dans son rapport plus qu'ambigu, le Service déclare que la vétuste Académie n'est plus vraiment sécuritaire tout en précisant qu'elle ne constitue pas non plus de danger pour 
le public ${ }^{19}$. Cette mauvaise publicité, bien importune, met un terme à la résistance de Murphy et entraîne la fermeture de l'établissement. Pas pour longtemps! Dès le 7 septembre suivant, après des modifications mineures, la "nouvelle " Académie de musique rouvre ses portes. Elle est désormais dirigée par Sparrow et Jacobs. Le Trust contrôle ainsi les trois plus importantes scènes montréalaises. Seul lui échappe le thêâtre Français qui, à vrai dire, ne présente pas de grands dangers et ne peut être tenu pour un rival sérieux dans les circonstances.

Fort de sa situation de quasi-monopole, le Trust entreprend de réformer le marché local et impose une série de mesures à ses deux agents montréalais. La plus étonnante et sûrement la plus maladroite d'entre elles est d'exclure Montréal - et tout le Québec - du circuit des tournées les plus prestigieuses d'Amérique - le High Road - , celles qui mobilisent les plus grandes vedettes américaines et internationales de l'heure et qui présentent les succès les plus percutants de Broadway (généralement adaptés de ceux de Londres et de Paris). Cette exclusion soulève la colère des anglophones de Montréal qui, pour une rare fois de leur histoire, manifestent quelques vélléités d'autonomie culturelle ${ }^{20}$. La décision du Trust place Sparrow et Jacobs dans une situation très inconfortable. Mais il $\mathrm{y}$ a pire, car s'ils contrôlent désormais trois théâtres, ils n'ont plus aucun accès aux spectacles de la catégorie supérieure. Ils doivent donc se rabattre sur les spectacles de catégories moyenne et inférieure Middle of the Road et Low Road. L'Académie de musique, dont les vicissitudes n'ont pas entamé le prestige, est d'emblée recyclée en théâtre intermédiaire, accueillant des comédies musicales et des drames de bon aloi. Mais que faire du Queen's et du Royal? Une scène suffit à combler les besoins du marché intermédiaire et hisser le Queen's à ce niveau serait suicidaire. Par ailleurs, le reléguer au niveau inférieur, celui du non legitimate et des variétés, le place en concurrence directe avec le théâtre Royal qui a déjà à composer avec la présence d'une multitude de petits établissements de variétés et de vaudeville américain, ainsi qu'avec les muséums. Mais Sparrow et Jacobs n'ont pas le choix! Ils se résignent à maintenir en activité les deux établissements et à y offrir le même type de divertissement. 
Cela les place dans une situation pour le moins paradoxale. Le Trust, qui les a progressivement amenés à contrôler les trois grands thêtres et à assurer leur mainmise sur la ville, les confine à un secteur - celui des variétés et du non legitimate — dans lequel il refuse lui-même de s'engager. Tout se passe comme si le Trust avait décidé qu'un seul thêâtre - l'Académie de musique - pouvait satisfaire les besoins thêâtraux des Montréalais. Peu lui importe alors l'embarras dans lequel ce choix place ses propres agents! Cette double erreur de jugement — à l'égard du marché montréalais et à l'égard de ses alliés - lui coûtera fort cher. Mais au demeurant, Sparrow et Jacobs ne peuvent compter que sur eux-mêmes pour assurer l'existence de leurs deux thêatres. Ils mobilisent toutes leurs relations et frappent à toutes les portes possibles pour dénicher des spectacles dignes de leurs ambitions. Mais vu la rareté des productions disponibles, ils en viennent à accepter à peu près n'importe quoi. Le cinéma est, dans les circonstances et en dépit de ses faiblesses, un moindre mal. D'autant plus que, de l'avis de la critique, le marché est si malade que les intermèdes cinématographiques sautillants et embrouillés valent souvent mieux que ce qui les précède et qui les suit.

La cause des deux alliés du Trust est rendue plus délicate encore en raison des initiatives de William Phillips, le nouveau propriétaire du théâtre Français. Déterminé, lui aussi, à mener la vie dure au Trust, qui a ruiné ses ambitions aux États-Unis, Phillips espère bien faire de cet immense théâtre, à la carrière en dents de scie, une scène indépendante susceptible d'attirer tous ceux - artistes et spectateurs - que rebutent les tactiques et l'arrogance du Trust. Parce qu'il n'a évidemment pas accès aux productions de celui-ci, Phillips doit faire affaire avec d'autres petits indépendants qui, à mesure que le temps passe, deviennent de plus en plus rares. Pour éviter l'asphyxie, il envisage un moment d'installer une troupe à demeure dans son théâtre. Mais la tentative tourne court. Les scènes anglophones de la ville - car en dépit de son nom, le Français est d'abord un théâtre anglophone - ont depuis longtemps déjà adopté le rythme hebdomadaire qu'imposent les tournées. La troupe médiocre recrutée par Phillips n'est évidemment pas en mesure de soutenir pareille cadence et Phillips doit se résoudre à la 
congédier. Ne reste plus alors, pour lui, que le marché terriblement sollicité des variétés. Par la force des choses plus que par choix, il se trouve ainsi à rivaliser avec le Queen's et le Royal dont la situation demeure précaire. Il ne s'en relèvera pas!

Bien installé dans les "grands" théâtres, qu'il ne quittera désormais que pour de brèves périodes, le cinéma entreprend alors sa pénétration vers les scènes moins importantes où il aurait dû faire ses premières armes. On le voit attirer les foules au parc Sohmer, puis à l'Eden Museum - un autre muséum du boulevard Saint-Laurent, situé au sous-sol du MonumentNational, puis au Monument-National lui-même ${ }^{21}$. C'est alors que survient le grand moment de cette jeune carrière. Le 22 mai 1897, 11 mois après Pupier et Minier, Félix Mesguich, un autre Français représentant les intérêts des frères Lumière, réinvestit la petite salle de l'ex-Gaiety Museum \& Theatorium et ancien Palace Theatre du boulevard Saint-Laurent. Et pendant au moins quatre mois, moyennant quelques interruptions, Montréal peut s'enorgueillir de posséder une véritable salle de. cinéma, une salle exclusivement consacrée au cinéma. Du statut d'attraction secondaire sur des scènes majeures, le cinéma devient ainsi l'attraction unique d'une scène mineure de la ville. Sa voie est tracée.

La conjoncture particulière à Montréal continuera d'infléchir la destinée du cinéma. Il en résultera une histoire étonnante et à bien des égards incomparable dont on commence à peine à prendre toute la mesure. Si le cinéma des premiers temps qu'on consomme ici vient très majoritairement d'ailleurs, la façon qu'on a de le voir, de l'organiser et de le présenter est indéniablement d'ici.

Collège de Valleyfield

\section{NOTES}

1 Grâce, principalement, aux travaux que le GRAFICS, Groupe de recherche sur l'avènement et la formation des institutions cinématographique et scénique, mène sur la question depuis juin 1994.

2 Ce qui n'exclut pas que, lui aussi, souffrira, à l'occasion et parfois gravement, de la désaffection du public. 
3 Ces six hommes, tous américains, sont Charles et Daniel Frohman de New York, Samuel F. Nirdlinger et John Frederick Zimmerman de Chicago, ainsi que Marc Klaw et Abraham Erlanger, également de New York. Ces deux derniers ne possédaient pas de théâtres, mais étaient les plus grands producteurs et distributeurs du continent.

4 Il n'existe pas de traduction française vraiment satisfaisante pour ce genre. Il s'agit, en général, de spectacle à texte - avec auteur - (tragédie, drame, mélodrame, comédie, comédie musicale, théâtre lyrique) nécessitant une imposante distribution et constitué d'actes successifs. Le legitimate englobe le thêâtre dit de répertoire et inclut également tout le théâtre contemporain. Il correspond, globalement, à ce qu'on pourrait qualifier de genres majeurs.

5 Le non legitimate peut être assimilé aux genres mineurs et aux spectacles les plus populaires tels que les gros mélodrames, et, surtout, les spectacles de variétés ou Variety Shows. Ces derniers consistent d'ordinaire en une succession de numéros hétéroclites qui vont du tour de magie aux monologues, en passant par les chanteurs de genre, les numéros de danse, d'acrobatie, de mime ou d'animaux savants, les courtes comédies musicales ou non - , farces ou drames en un acte. Le vaudeville américain, quoique plus structuré que le spectacle de variétés habituel, est sensiblement identique, mais s'adresse à une clientèle plus familiale et exclut donc, en principe, tout numéro à caractère érotique.

6 On a vu des artistes, tels que James O'Neill, le père du dramaturge Eugene O'Neill, consacrer toute une carrière à l'interprétation d'un ou de deux rôles à succès (en l'occurrence ceux d'Edmond Dantes dans Le Comte de Monte Cristo ou de d'Artagnan dans Les Trois Mousquetaires).

7 Repris à Montréal par le journal The Gazette (4 mai 1895, p.12).

8 Dans l'organisation de leurs tournées continentales, les troupes prévoyaient toujours un arrêt minimal d'une semaine à Montréal. Les grands théâtres de la ville changeaient ainsi de programme tous les lundis.

9 L'Académie de musique se trouvait juste au nord de Sainte-Catherine, à un endroit aujourd'hui occupé par le magasin Eaton du centre-ville. Quant au théâtre Royal, qui est alors sur son déclin, il se trouvait rue Côté, à proximité de l'actuel Palais des congrès de Montréal.

10 Le bâtiment où eut lieu cette première historique existe encore et est demeuré à peu près inchangé. Il s'agit de l'édifice Robillard, du 972-976 Saint-Laurent, au coin nord-est de Viger et de Saint-Laurent.

11 Le muséum comporte généralement deux sections et deux espaces distincts: la salle de spectacles, limitée, le plus souvent, à quelques centaines de sièges tout au plus et munie d'une scène rudimentaire aux dimensions restreintes, et la salle d'exposition. C'est cette dernière section qui justifie l'appellation de l'établissement et lui confere des qualités vaguement scientifiques. Le Gaiety Museum and Theatorium, où Louis Pupier et Louis Minier réalisèrent leur première démonstration du cinématographe, avait abrité un musée de cire.

12 Tiré d'un article anonyme de La Presse intitulé simplement "Le Cinématographe", et publié le lundi 29 juin 1896, p. 1.

13 Situé à l'ouest du Vieux Port actuel, le parc Sohmer était un parc d'attraction familiale qui possédait également une petite salle de spectacles.

14 Publicité du théâtre Royal parue dans La Presse du samedi 26 septembre 1896, p. 15. Les mentions subséquentes du journal ne font que reprendre l'information sans la préciser.

15 Situé là où se trouve maintenant la discothèque Metropolis, sur SainteCatherine, à proximité du boulevard Saint-Laurent.

16 Publicité de La Presse, lundi 14 décembre 1896, p. 7. 
17 Annonce parue dans La Presse du samedi 16 janvier 1897 (p. 14), puis du mardi 19 janvier (p. 7). Selon toute vraisemblance, les premières projections débutèrent le lundi 11 janvier puisque le communiqué du 16 précisait que "le cinémascope rest[e] une autre semaine, mais les vues [sont] entièrement changées".

18 Les trois théâtres jouaient, à cette époque, en matinée et en soirée, six jours par semaine. Ils faisaient relâche le dimanche. Le théâtre Royal contenait 1500 sièges, le Queen's près de 2000 et le théâtre Français 3000.

19 On trouve le détail de cette affaire ténébreuse dans un article publié par The Gazette, le 9 mars 1896, p. 3.

20 Constatant leur dépendance, ils se prirent en main et résolurent de construire d'eux-mêmes un théâtre haut de gamme afin d'accueillir, comme ils l'avaient toujours fait, les vedettes internationales de l'époque. Leur initiative résulta, en 1898, dans la construction du Her Majesty's, rue Guy, un peu au nord de Sherbrooke. Pendant près de 40 ans, ce théâtre perpétua la tradition d'excellence instaurée au préalable par l'Académie de musique.

21 Le Radioscope, avec vues coloriées, triomphe au parc Sohmer dès le 23 mai 1897 (voir "Parc Sohmer", anonyme, La Presse, lundi 24 mai 1897, p. 2), un jour plus tard que le Feriscope qui attire les foules à l'Eden (avec, entre autres, le combat entre Corbett et Courtney). Le Monument-National présentera, quant à lui, le combat entre Corbett et Fitzsimmons à compter du 14 novembre. 